\title{
PRINCIPLES OF THREE DIMENSIONAL SPATIAL MULTIPLEXING IN WIRELESS COMMUNICATIONS
}

\author{
Oleksandr V. Mazurenko, Yevhenii A. Yakornov \\ National Technical University of Ukraine "KPI", Kyiv, Ukraine
}

This paper presents a comparative analysis of the multiplexing principles, allowing increasing the technical efficiency of radio resource usage based on the three-dimensional signals spatial separation in a spherical coordinate system. These methods allow creating spatial multichannel communications between certain points (areas) of the space through the radiators and signal processing system of wireless base station. Three-dimensional spatial selectivity in spatial matched filtration is provided by the physical processes of the focusing and the differentiation signals by their front curvature, and in the spatial correlation signal processing - by orthogonality of spreading sequences, their reusing by cyclic shift and synchronization, depending on the distance from the base to the mobile station.

\section{Introduction}

Capacity increasing of data transmission system in some spatial volume, frequency band and time interval is the most important task in wireless communication technique. The most advanced techniques that solve the problem mentioned above is spatial multiplexing system based on directional antenna with sectorial radiation [1] and MIMO system technology [2]. The devices that realized these techniques provide signals transmission in "direction-to-direction" mode between two mobile stations (MS) through base station (BS) antenna system, since the spatial signal division is provided in two angular spatial coordinates, - azimuth and elevation, - in three-dimensional space of spherical coordinates. Thus, MS located on the line between BS and currently serviced MS should wait their queue or split the used frequency spectrum resource. That essentially is a conflict situation. The key solution of this conflict is increasing of radio frequency resource usage efficiency by including an additional dimension of distance in spatial signal division process. Then, spatial signal transmission is proceeded in "point-to-point" mode, thus signal division is provided in all three spatial dimensions of spherical coordinates.

This new spatial multiplexing method implementation result leads to dividing of primal service area to several sub areas that serviced by primal radio unit or BS, but not its own equipment as usual if it is regarded on the plane, as on fig.1. Equipment reducing is another consequence of the implementation.

Requires attention the fact that MIMO technique with space-time coding (STC), in a some sense, is able to provide three-dimensional spatial signal multiplexing, whereas it's based on adaptive adjustment to impulse response of spatial channel between two certain points. However, appears, that there are a lot of these spatial points with identical impulse response. In addition, orthogonality between large number (6-8) of spatial channels is not provided on high level, that reduces their productivity [3]. This productivity reduction appears due to MIMO STC technique operation nature based on system independent spatial channelizing principles $[4, \S 1]$.

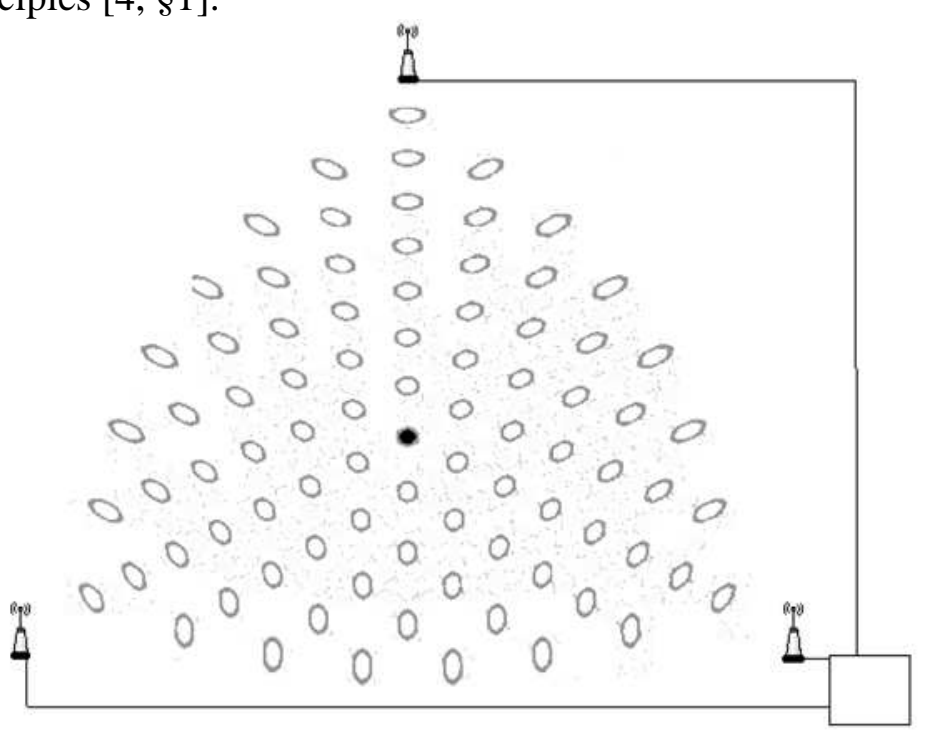

Fig.1. Three-dimensional spatial multiplexing

Also, another ways of providing of signal division by distance dimension exist, then wireless communication is regarded on the plane which serves as a dividing factor and it is irradiated by directive antennas from points on some height above this plane at different azimuth or elevation angles. However, such ways of pseudo three-dimensional spatial signals multiplexing achieving requires additional expenses for lifting directive antennas at sufficiently high altitude and providing their functioning in adverse weather conditions. At 
the same time, problem of reflected signals and signals that passed the target point, which further act as interference to neighboring MS is not solved.

Taking into account all the problems that appear during arranging of pseudo three-dimensional spatial signals multiplexing, it is required to find such signal processing principles that allow implementing the real three-dimensional spatial signals division without additional expenses for infrastructure and with system dependent spatial channelizing condition fulfillment. System dependent spatial channelizing is a channel creation process that excludes influence of signal multipath propagation effects $[4, \S 1]$.

If criteria mentioned above are considered from optimal filtration theory viewpoint [5], then the necessary solution based on spatial matched filtration (SMF) can be implemented by means of focused antenna arrays (FAA) [6] which use "optical" focusing and signal division by it electromagnetic wave front curvature processes. Also there is a solution based on spatial correlation signal processing (SCSP) [7] that uses orthogonality of spreading sequences and their set reusing possibility by cyclic shift [8], and special synchronization procedures that depend on distance between MS and BS. These solutions can provide signal division and multiplexing in all three spatial dimensions of spherical coordinates.

This paper is devoted to the comparative analysis of the principles of multiplexing based on solutions mentioned above.

\section{Multiplexing based on spatial matched filtration}

This three-dimensional spatial multiplexing method operating principle based on spatial channelizing features that provided by specialized FAA [6], that uses "optical" focusing and signal division by it electromagnetic wave front curvature processes for signals transmitting and receiving respectively. These features fulfill all requirements for controlled formation of signal power spatial distribution (SPSD) in all three dimensions of spherical coordinates. Herewith, SPSD by distance depends as on affect of attenuation from propagation, as on signals time/phase distribution adjusting at FAA elements.

Formation of spatial channels requires one FAA that installed on BS for each cannel, according to physical principles that underlying the operation of FAA. MS may use directional antenna, which not having the FAA properties, because BS FAA forms certain service area exclusively for target MS. But MS antenna radiation should not interfere with the neighboring MS. In such case, transmit and receive channels must be separated by frequency in order to separate the signals that transmitted by one MS and received by others. In case, when transmit and receive channels are located in the same frequency band and in the same time interval, then MS must use FAA as BS. This spatial multiplexer channels distribution is described by mathematical apparatus of FAA SPSD synthesizing which takes into account location (spatial distribution) and signals amplitude-phase distributions of FAA elements that presented in details at [6].

According to [6], practical realization of spatial signal division by distance for narrowband signals is possible in millimeter waves range. For such principle realization in lower frequency band with all criteria of necessary spatial channels orthogonality fulfillment need to change transmitting signal "sinusoidal" carrier for ultra wideband impulse.

FAA angular spatial resolution is greater for two orders than their resolution by distance. Resolution by distance between two spatial channels can be really achieved in range of 10-30 dB. Resolution by angular dimensions and by distance defines focal area transverse size and it "depth" (focal depth) respectively.

Focal depth by $-3 \mathrm{~dB}$ signal level calculated from [6]:

$$
\Delta R=\frac{2 \lambda\left(R_{F} \cos \left(\theta_{F}\right)\right)^{2}}{D^{2}-\left(\lambda R_{F} \cos \left(\theta_{F}\right) / D\right)^{2}},
$$

where $R_{F}, \theta_{F}$ - focal point distance and angular coordinate, $D$ - maximum linear size of FAA, $\lambda$ - wavelength.

Circular FAA with diameter $D=2 \mathrm{~m}$, operational frequency $F_{\mathrm{C}}=120 \mathrm{GHz}$ SPSD cross section in azimuthal plane with take into account signal attenuation by propagation shown on fig. 2 .

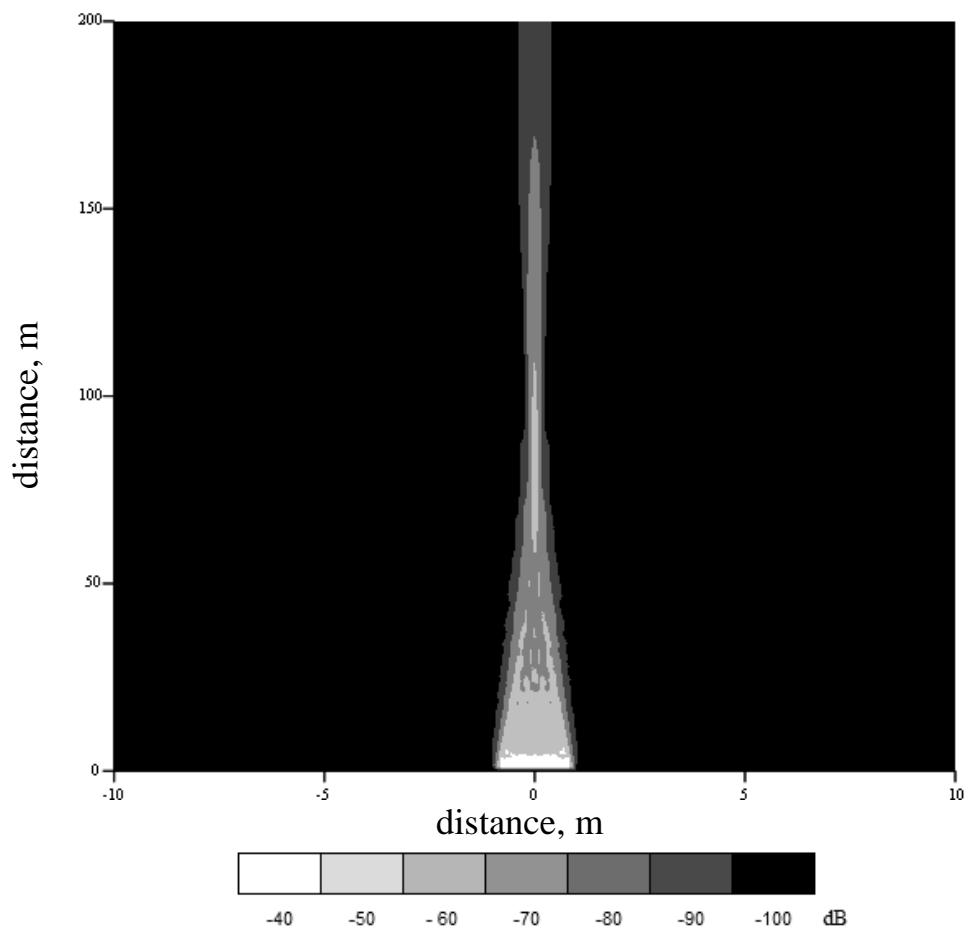

Fig.2. SPSD generated by FAA 
SPSD generated by FAA that shown on fig. 2 describes spatial selectivity of single channel of threedimensional spatial multiplexer based on SMF.

Spatial multiplexer is based on SMF and consists of nine circular FAA with diameter $D=2 \mathrm{~m}$, operational frequency $F_{\mathrm{C}}=120 \mathrm{GHz}$ channels distribution / selectivity by distance without and with take into account signal attenuation by propagation shown on fig.3.

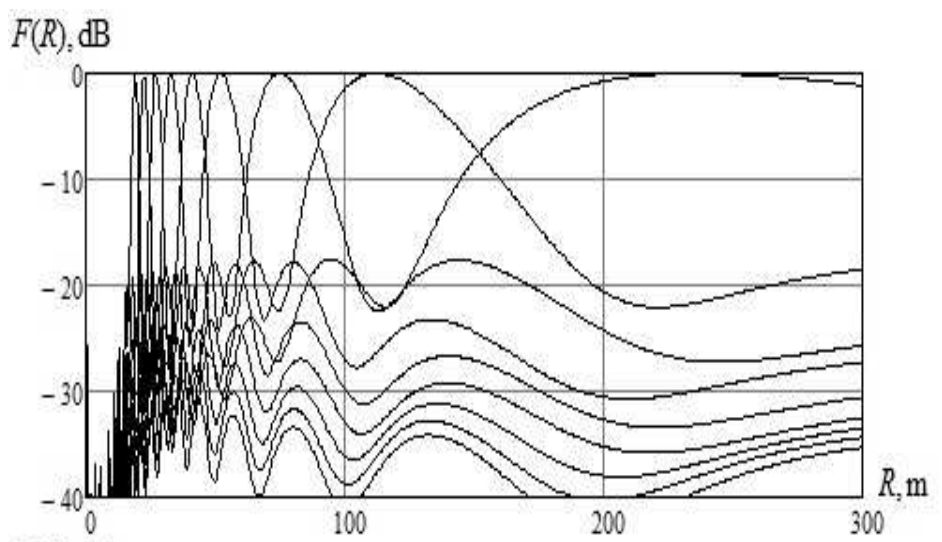

$F(R), \mathrm{dB}$

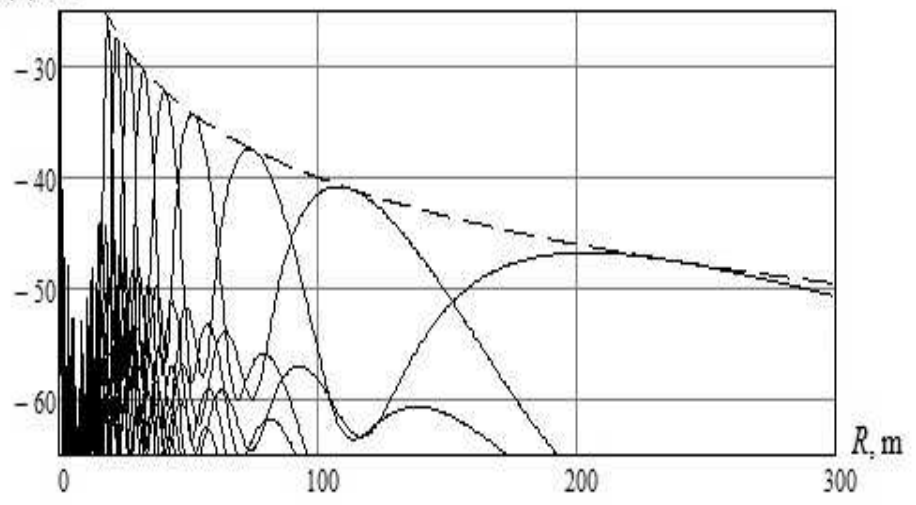

Fig.3. Spatial multiplexer based on SMF channels distribution / selectivity by distance

As shown on fig.3, selectivity by distance is decreased with its dimension increasing, thus specific signal power concentration in nearest and more distant areas that act as service sub areas is different and tends to decrease. Also, advisable that the maximum signal levels in nearest and more distant spatial channels difference due to signal attenuation by propagation not to exceed FAA adjacent spatial channel selectivity [6]. Physical processes that underlying the operation of FAA restrict its maximum operation radius by hyperfocus distance

$$
R_{\mathrm{HF}}=D^{2} / \lambda,
$$

where three-dimensional spatial selectivity still possible.

Taking into account all the information mentioned above, physically real three-dimensional spatial sig- nals division provided by FAA is sophisticated process which have essential but clear restrictions. Thus, spatial multiplexer based on FAA realization is complicated and expensive. According to [6], these restrictions are the result of using of "sinusoidal" carrier for signal transmission. Changing transmitting signal "sinusoidal" carrier to ultra wideband periodic impulse or baseband signal spectrum spreading leads to spatial resolution / selectivity and hyperfocus distance increasing equally to spreading spectrum ratio [6].

These FAA restrictions can be avoided by using another signal processing method - correlation signal processing, especially that based on spatio-temporal principles [7].

\section{Multiplexing based on spatial correlation signal processing}

This multiplexing technique has become as evolution of SMF method and modification of multiplexing system based on code division principles by altering temporal operation principle basis to spatiotemporal. Its distinguishing feature is the possibility of several spatial channel forming by single directive antenna with angular spatial selectivity by means of changing transmitting signal "sinusoidal" baseband carrier to orthogonal spreading sequence (OSS) set baseband basis. Where selectivity by distance is provided by SCSP. Respectively, spatial signal division by distance is realized not physically but virtually by means of SCSP. Spatial resolution by distance dependent not on antenna system physical parameters but on OSS virtual parameters that also is SCPS distinguishing advantage which removes restrictions on used frequency range.

Spatial channel capacity increasing is provided by OSS set reusing by its cyclic shift that presented in details at [8].

Special synchronization procedures that provide synchronous signal transmission mode in "up" and "down" direct spread spectrum system channels are necessary for this technique operation providing. Thus, MS system clock source initial phase dependent on distance from its location to BS for "primal" OSS set and for reused set is dependent with take into account respective time shift equal to OSS cyclic shift time interval.

Spatial multiplexer consists of sectorial antenna and SCSP unit that run with 32 symbols OSS set, OSS reusing rate $N=2$, operating frequency $F_{\mathrm{C}}=$ $2,4 \mathrm{GHz}$ and symbol rate -10 Mchip per second channels distribution / selectivity by distance with- 
out and with take into account signal attenuation by propagation shown on fig. 4 .

As shown on fig.4, spatial channels orthogonality is provided by OSS that generated these channels initial phase delay for a value when OSS set autocorrelation $(\mathrm{ACF})$ and cross-correlation functions (CCF) magnitude equal to zero.

Shown on fig.4, spatial selectivity was performed when MS and BS clock source has the same initial phase (as they are synchronized in some point by certain signal) as they not care about distance between each other, but each BS correlators clock source initial phase has phase shift equal to signal propagation time from respective MS to BS.

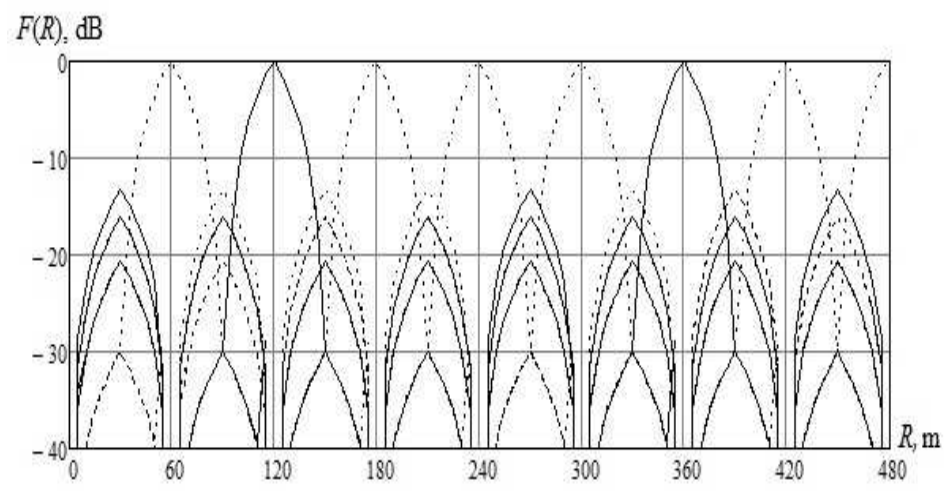

$F(R), \mathrm{dB}$

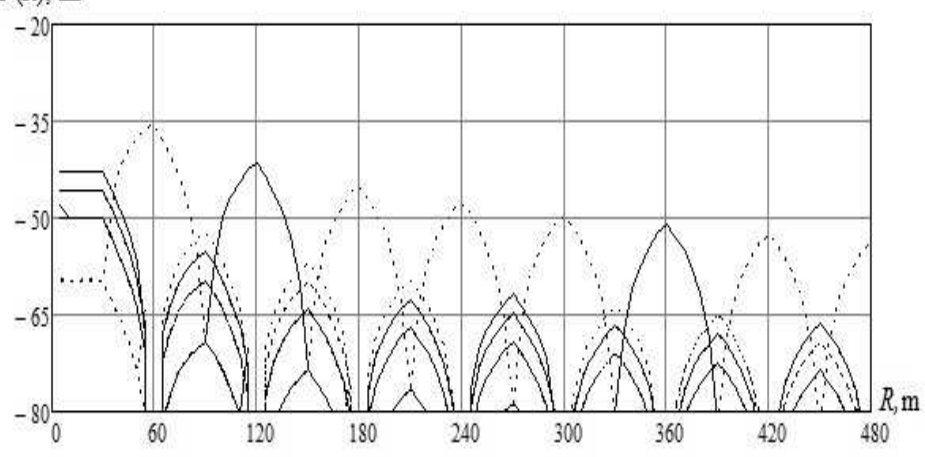

Fig. 4. Spatial multiplexer based on SCSP channels distribution / selectivity by distance

Consider the synchronization principles required for this multiplexing method operation. For the new system synchronization principles synthesis, define the value of the signals initial phase $\tau_{0}$, that transmitted $\uparrow$ and received $\downarrow$ by MS and BS in current code division multiple access wireless communication system [9] not taking into account time delays for signal processing and it passing through BS and MS receiver/transmitter feeder lines.

BS transmits pilot signal with initial phase $\tau_{0}{ }^{\mathrm{BS}} \uparrow=0$, then it is propagated to MS through path of length $d$ with speed of light $c$ and received with initial phase relative to BS $\tau_{0}{ }^{\mathrm{MS}} \downarrow=d / c$. MS sets its own clock source initial phase equal to received pilot signal phase and then transmit service signal with the same initial phase $\tau_{0}{ }^{\mathrm{MS}} \uparrow=d / c$. Then, BS receives signal with initial phase $\tau_{0}{ }^{\mathrm{BS}} \downarrow=2 d / c$, in other words, BS receives from MS the same pilot signal with initial phase difference $\Delta \tau_{0}=2 d / c$.

Now it became clear, that BS receives signals from MSs asynchronously due to different distance between each other. First channel signal from MS1 and second channel signal from MS2 are asynchronously received by BS and became co-channel interference for each other according to their channels source OSSs CCF magnitude in value of difference of signal time propagation due to different specific location of MSs relatively to BS.

Also, difference between BS and MS established clock source initial phase can be determined if MS clock source is adjusted at location on distance $d$ ' from BS and then after MS will become to BS location their clock source initial phase difference is determined as equal to $\Delta \tau_{0}=d^{\prime} / c$.

Thus, to provide the condition of synchronous signal receiving at $\mathrm{BS}$ it is necessary that signals from all MS are became to BS with initial phase $\tau_{0}{ }^{\mathrm{BS}} \downarrow=0$ or with such $\tau_{0}{ }^{\mathrm{CCF} 0}$ value when CCF magnitude of all OSSs is become zero. Then, before signal transmission every MS must shift its clock source initial phase by value

$$
\begin{gathered}
\tau_{0}{ }^{\mathrm{MS}} \uparrow=-d / c=T-d / c, \\
\tau_{0}{ }^{\mathrm{MS}} \uparrow=\tau_{0}{ }^{\mathrm{CCF} 0}-d / c=T+\tau_{0}{ }^{\mathrm{CCF} 0}-d / c,
\end{gathered}
$$

$T$-OSS period.

MSs shift their clock source initial phase to such value that their real locations determined by $\mathrm{BS}$ as usual become virtual from which $\mathrm{BS}$ receive signals synchronously.

If principle of channel capacity increasing by OSS set reusing with its initial phase shift to value $\tau_{0}{ }^{\mathrm{CCF} 0}$ is used, in that case all MSs that operate with primal OSS set must shift transmitted signals initial phase to value

$$
\tau_{0}^{\mathrm{MS}} \uparrow=-d / c=T-d / c,
$$

then these signals are received by BS coherently. And all MSs that operate with reused OSS set by its initial phase shift to value $\tau_{0}{ }^{\text {CCF0 }}$ must shift transmitted signals initial phase to value

$$
\tau_{0}{ }^{\mathrm{MS}} \uparrow=\tau_{0}{ }^{\mathrm{CCF} 0}-d / c=T+\tau_{0}{ }^{\mathrm{CCF} 0}-d / c,
$$

then these signals are received by BS coherently if BS shift corresponding correlators clock source initial phases to value $\Delta \tau_{0}=\tau_{0}{ }^{\text {CCF0 }}$.

Channel capacity in direction from $\mathrm{BS}$ to $\mathrm{MS}$ could be increased as for reverse direction case with 
take into account such a fact that MSs receive signals from BS always coherently if their clock sources initial phases are adjusted by BS pilot signal. Thus, in that case, necessary shift transmitted by BS signals and MSs correlator clock source initial phases simultaneously and at same value of $\Delta \tau_{0}$ $=\tau_{0}{ }^{\mathrm{CCF} 0}$.

When OSS set is reused, necessary to follow the condition of primal and reused OSS sets (with shifted initial phase to value $\tau_{0}{ }^{\mathrm{CCF} 0}$ ) ACF and CCF "zero positions" equality, then reusing rate $N$ and initial phase shift $\tau_{0}{ }^{\mathrm{CCF} 0}$ are related as

$$
\Delta \tau_{0}{ }^{\mathrm{CCF} 0}=T / N \text {, where } N=2^{n}, n \in \mathbf{Z} \text {. }
$$

All OSS sets "absolute" orthogonality are provided only in the case when condition revealed above is performed.

Therefore, define that essence of new synchronization principle is to establish such temporal relations between the initial phases of signals from different MSs at which some channel signal ACF main lobe coincides with other channels signal CCF "zero points".

Summarizing all the definitions mentioned above, such synchronization and code division channel distribution systems can be built which allow to decrease of signals initial phase adjusting range from $[0, T]$ to $\left[0, \Delta \tau_{0}{ }^{\mathrm{CCF} 0}\right]$ and to divide primal service area into $N$ service sub areas that are maintained by one BS radio unit.

Thus, if there is such OSS set with reusing rate $N$ and service area with radius

$$
R=N \cdot T \cdot c,
$$

then this service area is divided into $N$ service sub areas with external radius

$$
R=k \cdot T \cdot c
$$

and internal radius

$$
R=(k-1) \cdot T \cdot c,
$$

where $k \in(1, N)$.

All MSs are attributed to service sub area with index $k$ where they are located and use primal or reused OSS set allocated for related sub area for spatial channelizing.

Synchronization starts as usual in code division multiple access wireless communication systems BS transmit pilot signal and MS is configured for operating by this signal. After, distance between BS and MS is determined by transmitting of service signals from BS to MS and in reverse directions and estimation of overall propagation duration $\Delta \tau_{0}=2 \mathrm{~d} / \mathrm{c}$ which allows computing final value $d$. Information about propagation duration and distance to its location is sent to corresponding MS. Then, MS by the value of $d$ determine attribution index

$$
k=\lfloor R / d\rfloor,
$$

where $L\rfloor$ rounding down operator. Thus, nearest to BS service sub area has index $N$, and farthest -1 .

Next step is allocation of some channel capacity to MS located in $k$-th service sub area for signal transmission in direction from BS to MS that, essentially, is determination of MS receiver clock source initial phase taking into account the condition of necessary to ensure clock source initial phase equivalence to distance between MS and BS for providing signals for MSs located in different sub areas spatial division.

OSS sets ACF main lobes allocation in the space by distance with maximum density can be done as one main lobe for one service sub area, since, sub area and SPSD/ACF main lobe by $-3 \mathrm{~dB}$ level sizes by distance as usual not equal that is caused by OSS correlation properties but rather OSS symbols number and reusing rate inequality

Search and study of OSS sets that could be reused are performed easily by construction of the special correlation fields (3D surfaces) in MATLAB that consist of multiple correlation function arranged in order $\mathrm{ACF}_{1}, \mathrm{CCF}_{12}, \ldots, \mathrm{CCF}_{1 \mathrm{~N}}, \mathrm{ACF}_{2}$, $\mathrm{CCF}_{21}, \quad \mathrm{CCF}_{23}, \quad \ldots, \quad \mathrm{CCF}_{2 \mathrm{~N}}, \quad \mathrm{ACF}_{\mathrm{N}}$, $\mathrm{CCF}_{\mathrm{N} 1}, \mathrm{CCF}_{\mathrm{N} 2}, \ldots, \mathrm{CCF}_{\mathrm{NN}-1}$ and their position indicated on abscissa $(X)$ axis, time shifts indicated on ordinates $(\mathrm{Y})$ axis, functions magnitude indicated on $\mathrm{Z}$ axis, as shown on fig.5.

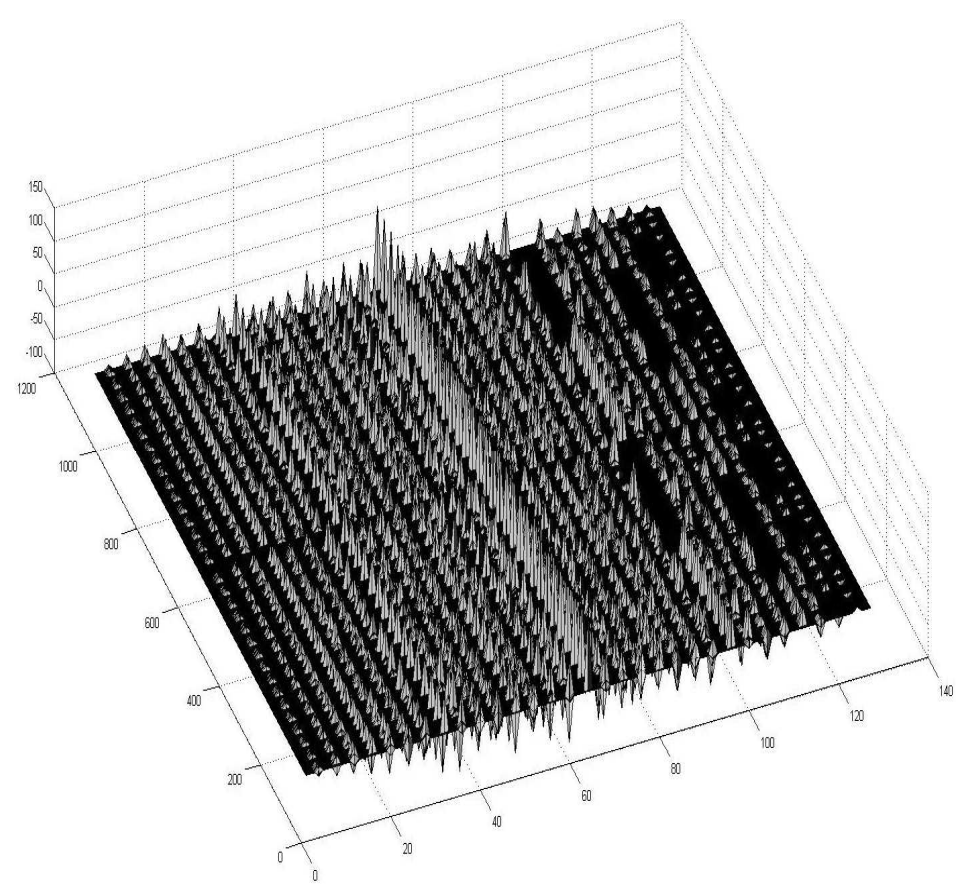

Fig. 5. 64 symbols complementary OSS sets correlation function field 
Studies have shown that this multiplexing method with using of common modulation techniques can be realized by using of only binary OSS such as complementary (C-) sequences or zero correlation zone (ZCZ/Z-) sequences [10].

The problem of finding OSS sets with similar features, as shown on fig.5, still remains an actual, because existing OSS sets did not allow achieving reuse rate more than 4 .

\section{Conclusions}

Three-dimensional spatial multiplexing is based on signals division between MSs that spatially separated by angular coordinates and distance via special signal processing systems which operation based on SMF and SCSP principles according to the theory of optimal filtering.

Comparing these principles it is possible to make the following conclusions.

Spatial multiplexing based on SMF can be realized with selectivity by distance of 10-30 dB and by angular coordinates of 30-50 dB between adjusted spatial channels by means of FAA. One FAA is able to form one spatial channel. Three-dimensional spatial selectivity can be achieved in certain range of distances - from BS to hyperfocus distance. FAA capability can be enhanced by changing transmitting signal "sinusoidal" carrier to ultra wideband periodic impulse or baseband signal spectrum spreading.

Configuration and construction of spatial multiplexers based on SCSP are more flexible than based on SMF because its selectivity more depends on channelization OSS features then on antenna features. But spatial signal division based on SCSP essentially is "virtual" unlike based on SMF which is physically natural.

Using of directed antennas for spatial signal division by angular coordinates and correlation signal processing for signal division by distance is the most optimal for this type of multiplexing.

Signal division by distance in this case is possible when special synchronization procedures are provided, at which OSS signals from all MSs are received by BS coherently.

Channel capacity is increased by reusing of OSS set that consists of complementary sequences and zero correlation zone sequences. Existing methods of OSS synthesis is able to create sets of reusing rate of 4 .
Therefore, three-dimensional spatial multiplexing is realized in two ways. The first one (SMF) implements the physically natural signals division, but expensive to implement and has structural constraints caused by the used frequency range and type of signal carrier. The second (SCSP) is more flexible and without structural constraints, but implements the essentially "virtual" signals division, that reducing channels security from unauthorized access. Also, further studies are necessary for improving of convenient OSS sets synthesis technique with purposes of spatial channel capacity increasing.

\section{References}

1. Ahmed El Zooghby. Smart antenna engineering. Artech House, 2005. 313 p.

2. Mohinder Jankiraman. Space-time codes and MIMO systems. - Artech House, 2004. 338 p.

3. Luis Garcia Ordonez. Performance Limits of Spatial Multiplexing MIMO Systems: Ph.D dissertation. Barcelona, Technical University of Catalonia, Spain. 2009. $228 \mathrm{p}$.

4. Honglin $\mathrm{Hu}$, Yan Zhang, Jijun Luo. Distributed Antenna Systems: open architecture for future wireless communications. - NY: Auerbach Pub., 2007. - 490p.

5. Korostelev A. A. Space-time theory of radio system. - M.: Radio i Svyaz, 1987. - 320p.

6. Oleksandr Mazurenko and Yevhenii Yakornov. Focused Arrays Beamforming, Behaviour of Electromagnetic Waves in Different Media and Structures, Prof. Ali Akdagli (Ed.), InTech, 2011. pp.419-440. DOI: 10.5772/16980.

7. Mazurenko O.V. Antenni reshitky z neliniynoyu prostorovoyu obrobkoyu poligarmonichnih ortogonal'nih signaliv [Antenna arrays with nonlinear spatial processing of polyharmonic orthogonal signals]. Zbirnik naukovih prats' VI KNU im.Tarasa Shevchenka, 2011, vol.32, pp. 35-42.

8. Mazurenko O.V., Yakornov E.A. Mobile communication system capacity increasing with spectrum spreading by means of space-time matched signal reception. 2011 21nd Int. Crimean Conf. "Microwave \& Telecommunication Technology" (CriMiCo'2011). Sevastopol, 2011, pp.404-405.

9. Valeri P. Ipatov. Spread Spectrum and CDMA: Principles and Applications. - Wiley. 2005. 400p.

10. Gepko I.A. Complementarnye i spectralno effectivnye kody $\mathrm{v}$ radiotekhnologiyah chetvertogo pokolenya [Complementary and spectrum-efficiency codes in radio technique of $4^{\text {th }}$ generation].. - K. : Zvyazok, 2008. 223p.

Received in final form October 11, 2013 